\title{
Solution of network localization problem with noisy distances and its convergence
}

\author{
Ananya Saha Buddhadeb Sau
}

\begin{abstract}
The network localization problem with convex and non-convex distance constraints may be modeled as a nonlinear optimization problem. The existing localization techniques are mainly based on convex optimization. In those techniques, the non-convex distance constraints are either ignored or relaxed into convex constraints for using the convex optimization methods like SDP, least square approximation, etc.. We propose a method to solve the nonlinear non-convex network localization problem with noisy distance measurements without any modification of constraints in the general model. We use the nonlinear Lagrangian technique for non-convex optimization to convert the problem to a root finding problem of a single variable continuous function. This problem is then solved using an iterative method. However, in each step of the iteration the computation of the functional value involves a finite mini-max problem (FMX). We use smoothing gradient method to fix the FMX problem. We also prove that the solution obtained from the proposed iterative method converges to the actual solution of the general localization problem. The proposed method obtains the solutions with a desired label of accuracy in real time.
\end{abstract}

Keyword Network localization technique, Localization with non-convex distances constraints, Localization with noisy distances, Applications of Lagrange optimization in localization, Mini-max optimization problem, Non-convex optimization.

\section{Introduction}

In recent technological advances, sensor networks are being adopted for collecting data from different hostile environments and monitoring them (Figure 11). A network may consists of sensor nodes, RFID readers, or members in a rescue team in a disaster management system, etc. Air pollution

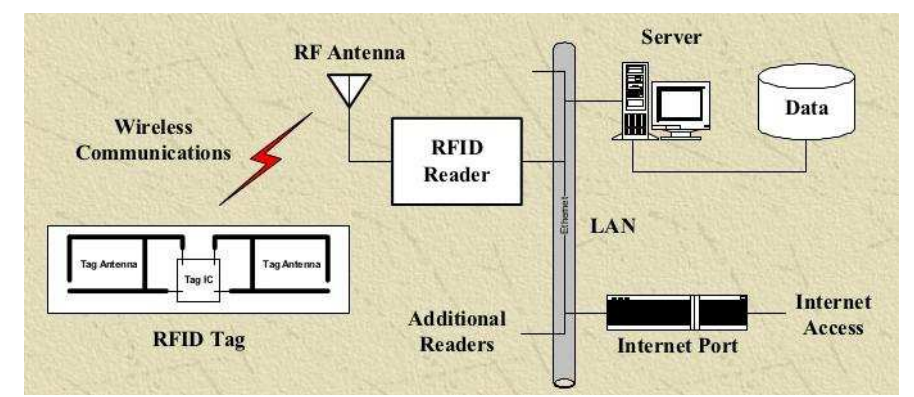

Figure 1: How does a network works

monitoring [20, forest fire detection [17, landslide detection [23], water quality detection [3], natural disasters prevention are some familiar field of applications in which sensor networks are useful. When a network is deployed in some region, the sensor nodes identify the events within their sensing ranges and transmit the collected information to the nodes within their communication ranges (a node within communication range of another node is called a neighbor). The location of an event 
can naively be estimated by the positions of the nodes identifying the event. Thus knowing the locations of the nodes are essential for properly monitoring the events. The objective of the network localization is to determine the node locations of a network using available distance information.

The GPS (Global Positioning System) 9] installation with each node of a network for finding its location is costly. Therefore the localization technique without using GPS needs research focus. Many researchers have proposed novel algorithms so far, for finding node positions using the information available from neighboring nodes. Distance measurements among neighboring nodes are popularly used for computing the node positions. These distances are measured by instruments embedded inside the nodes. It is practically difficult to exactly measure the distances among nodes even with existing sophisticated hard-wares. Thus the network localization with noisy distance measurements demands rigorous research. In the literature, there are several algorithms based on exact [29, 7] as well as noisy distances [12, 8]. Graph rigidity theory [29] and optimization theory are popularly used by researchers for developing localization algorithms [12, 8].

Any network may be represented by a distance graph (a graph with edge weights equal to the distances between the end points of the edge). The network localization problem with the graph model of the network is equivalent to the graph realization problem. In 1979, Saxe [29] proved that the problem of embedding graphs in the one dimensional space is NP-complete and in higher dimensional spaces it is NP-hard. Later Aspnes, Goldenberg and Yang [7] proved that the problem of finding a realization of a graph is an NP-hard problem even if it is known that the graph has unique realization.

During the last few decades, some variants of the general network localization problem have been solved. In [27, 26, 28, the localization problem with exact node distances has been discussed for wireless sensor networks; they used the ordering of nodes of the underlying network and graph rigidity property for localization. In distance-based network localization, the number of solutions of the network localization problem may be unique, finite or infinite (up to congruence). Testing the unique localizability of networks having exact distances among nodes has been discussed in [18, 6]. If a network is not uniquely localizable then it must have some nodes which may either be freely rotated with respect to some other nodes or reflected with respect to some edges. If some nodes of the network may be rotated then the number of solutions of the associated localization problem is infinite. If in the underlying graph of a network, a vertex (or a few vertices) may be reflected with respect to a set of neighbors that are almost co-linear then it is called a flip vertex of the network and this phenomenon in network localization is called a flip ambiguity. Analysis of flip ambiguity in network localization has been discussed in 19. To find unique localization of networks removing the flip ambiguity of nodes is essential.

In real field of applications, collecting the exact distances among adjacent pair of nodes is almost impossible. Doherty et al. [12 formulated the localization problem with noisy distance as a nonconvex optimization problem. They excluded the non-convex constraints from the general problem to obtain a convex version of it and solved the problem using semi-definite programming (SDP) [12]. Biswas et al. 8 converted the same non-convex network localization problem to convex localization problem by using the relaxation technique and solved by SDP. To the best of our knowledge, solving the general problem is still a challenge. In this work we sort the challenge by solving the general nonlinear non-convex network localization problem using Lagrangian optimization. As far as we know this is the first approach for solving the network localization problem without any modification of constraints.

In our previous works [24, 25] (published in the proceedings of international conferences), we converted the nonlinear non-convex network localization problem to a root finding problem of a single variable continuous function $\phi\left(c_{0}\right), c_{0} \in \mathbb{R}$ (where $\left.\phi\left(c_{0}\right)=\psi\left(c_{0}\right)-1\right)$. We choose the standard bisection method for solving this root finding problem since the iterations in this method are guaranteed to converge to a root. The root finding problem inherently includes the finite mini-max problem which is NP-hard [11. We used the sequential quadratic programming [16, 31, 22, method to solve the finite mini-max problem. Using the sequential quadratic programming method, an approximate finite mini-max value of the function $\phi\left(c_{0}\right)$ was computed at $c_{0}$. Therefore in the iterative method, the sign of the function $\phi\left(c_{0}\right)$ was determined incorrectly due to the approximation. For instance, suppose at an iterative step, an approximate value of $\phi\left(c_{0}\right)$ is .0055 . But the actual value of the 
function is -.0000025 . In this case, the bisection method will consider the sign of the function positive though it is actually negative. Thus the correct interval containing the root was not determined in the bisection method due to approximation. This is a drawback of using the bisection method which is rectified in this paper.

A network localization problem may have different congruent solutions in the Euclidean space even if the distance information are collected from some practical field of application of WSN. The Euclidean space is unbounded. Therefore the congruent solutions of the localization problem may be anywhere in the Euclidean space. In our work, we constructed the root finding problem [24, 25] such that the estimated node positions of the network will be closer to the origin of the Euclidean Space with respect to some rectangular axis. We established that, we may always identify a compact region (i.e., a closed and bounded region) in the two dimensional Euclidean space containing the origin within which the localization problem must have a solution [24, 25.

In this paper, the construction of the root finding problem from the network localization problem is revised thoroughly. We develop an iterative method in light of the bisection method for finding a root of $\phi\left(c_{0}\right),\left(c_{0} \in \mathbb{R}\right)$. In each step of the iterative method it is required to determine whether the function $\phi\left(c_{0}\right)$ has values with opposite signs at the end points of a sub-interval of the interval identified in the previous iteration. We compute a tight bound for the function $\phi\left(c_{0}\right)$ at $c_{0}$ which depends on its approximately computed value in the iteration. Using these bounds and the monotonic non-increasing property of $\phi\left(c_{0}\right)$ we determine the required sub-interval in our method. In this way without computing the exact value of $\phi\left(c_{0}\right)$ we proceed for finding a solution of $\phi\left(c_{0}\right)$. We establish that the method converges to a solution of the network localization problem and the solutions of root finding problem may be achieved up to a desired label of accuracy within an acceptable number of iterations.

Organization of the paper: In Section 2, we present the general network localization problem with the convex and non-convex distance constraints. The construction of Lagrangian form of the network localization problem is given in Section 3 . In Section 4, we discuss the technique for solving the Lagrangian network localization problem. Convergence of the solution technique is analyzed in Section [5along with some instances of networks for which we implement the root finding method for finding a localization. We sketch an error analysis of the proposed method in Section 6 and conclude in Section 7.

\section{Network localization problem}

Let $\aleph$ be an ad-hoc network; $V$ is the set of nodes $(|V|=\nu)$ and $E$ is the set of communication links. The underlying graph $G_{\aleph}(V, E)$ is the grounded graph of $\aleph$. A realization of $G_{\aleph}(V, E)$ in $d$-space is a 1-1 mapping $f$ from the vertex set $V$ to $\mathbb{R}^{d}$. Two different realizations $f$ and $g$ of $G_{\aleph}$ are equivalent if for each edge $\{u, v\}$ in $E,\|f(u)-f(v)\|=\|g(u)-g(v)\|$ where, $\|$.$\| is the standard Euclidean norm$ in $\mathbb{R}^{d}$. $f$ and $g$ are congruent if the equality $\|f(u)-f(v)\|=\|g(u)-g(v)\|$ holds for each pair of vertices in $V$. A realization $f$ of $G_{\aleph}(V, E)$ in $d$-space is unique up to congruence if every realization $g$ equivalent to $f$ is congruent to $f$. If $f$ and $g$ are congruent to each other then $g$ can be obtained from $f$ by a suitable transformation of the coordinate system in $d$-space and recomputing $f$ according to the new coordinate system. To fix a coordinate system in a $d$-space, $d+1$ independent points are required with known positions. Therefore a uniquely realizable framework can be uniquely located in a $d$-dimensional space if we can fix $d+1$ points in the space. On the other hand, if $G_{\aleph}$ has two or more equivalent realizations which are non-congruent in a $d$-space then $G_{\aleph}$ is called ambiguously $d$-realizable.

A distance based localization algorithm determines the locations of nodes in a network by using known positions of anchors, if any, and a given set of inter-node distance measurements. Let $A=$ $\left\{u_{1}, u_{2}, \cdots, u_{m}\right\}$ be the the set of anchor nodes with known positions $\left\{a_{1}, a_{2}, \cdots, a_{m}\right\}$ and $B=$ $\left\{b_{1}, b_{2}, \cdots, b_{n}\right\}$ be the nodes with unknown positions $\left\{x_{1}, x_{2}, \cdots, x_{n}\right\}$. In this work, we find the positions of the nodes in $B$ assuming $m=0$, i.e., the network has no anchor node. The technique is equally applicable for networks with anchor vertices. Let $N$ be the set of all edges joining $x_{i}$ 's. Upper and lower bound on the exact length of an edge in $N$ joining $x_{i}$ and $x_{j}$ are denoted by $\bar{d}_{i j}$ 
and $\underline{d}_{i j}$. Let $\underline{D}=\left(\underline{d}_{i j}\right)_{n \times n}$ and $\bar{D}=\left(\bar{d}_{i j}\right)_{n \times n}$ be the matrices with $i j$-th entries $\left(\underline{d}_{i j}\right)$ and $\left(\bar{d}_{i j}\right)$ respectively. If two nodes $x_{i}$ and $x_{j}$ are not adjacent in the grounded graph $G_{\aleph}(V, E)$ then both the matrices have $i j$-th entry zero. Under the anchor free setting, the network localization problem may also be formulated as a nonlinear problem. The problem may formally be described as follows.

Problem 1. Given the edge set $N$ and the matrices $\underline{D}=\left(\underline{d}_{i j}\right)_{n \times n}, \bar{D}=\left(\bar{d}_{i j}\right)_{n \times n}$, of the grounded graph $G_{\aleph}(V, E)$ of a network $\aleph$ with a set $B$ of $n$ nodes with unknown positions,

$$
\begin{array}{ll}
\text { Find } & X=\left(x_{1}, x_{2}, \cdots, x_{n}\right) \\
\text { such that } & \underline{d}_{i j}^{2} \leq\left\|x_{i}-x_{j}\right\|^{2} \leq \bar{d}_{i j}^{2},\left\{b_{i}, b_{j}\right\} \in N
\end{array}
$$

If $X^{\prime}$ is an estimation for the unknown positions of nodes in $B$ obtained by solving Problem 1 every realization congruent to $X^{\prime}$ obtained by translating the coordinate system is also a solution to Problem 1 The re-computations of such solutions can be avoided by including a function $\min \sum_{i=1}^{n}\left\|x_{i}\right\|^{2}$ in the Problem 1 as the objective function. A solution to this optimization problem will minimize the sum of square distances of unknown nodes from the origin.

Let each $x_{i} \in \mathbb{R}^{d}$ then $x=\left(x_{1}, x_{2}, \cdots, x_{n}\right)$ is a point in $\mathbb{R}^{d n}$. Suppose $|N|=n_{0}$. For each edge $e_{k} \in N$, let $f_{k}: \mathbb{R}^{d n} \rightarrow \mathbb{R}$ be the function

$$
f_{k}(x)=\left\|x_{i}-x_{j}\right\|^{2}, \quad 1 \leq k \leq n_{0}
$$

where $e_{k}=\left\{b_{i}, b_{j}\right\}$. Let $\underline{d}_{k}=\underline{d}_{i j}$ and $\bar{d}_{k}=\bar{d}_{i j}$. Using these notations, Problem 1 can be rewritten as Problem 2 .

Problem 2. Given the matrices $\underline{D}=\left(\underline{d}_{k}\right)_{n \times n}, \bar{D}=\left(\bar{d}_{k}\right)_{n \times n}$ of the network $\aleph$ with a set $X$ of $n$ nodes with unknown positions. Find solutions of the nonlinear optimization problem

$$
\begin{array}{ll}
\text { Minimize } & f_{0}(x)=\sum_{i=1}^{n}\left\|x_{i}\right\|^{2} \\
\text { such that } & \underline{d}_{k}^{2} \leq f_{k}(x) \leq \bar{d}_{k}^{2}, \quad \forall 1 \leq k \leq n_{0}
\end{array}
$$

In Problem 2, each constraint $\underline{d}_{k}^{2} \leq f_{k}(x) \leq \bar{d}_{k}^{2}$ can be broken into two parts, namely, $f_{k}(x) \leq \bar{d}_{k}^{2}$ and $\underline{d}_{k}^{2} \leq f_{k}(x)$. For each $k\left(1 \leq k \leq n_{0}\right)$, if $y_{1}, y_{2} \in \mathbb{R}^{d n}$ satisfy $f_{k}(x) \leq \bar{d}_{k}^{2}$ then $\forall t \in[0,1]$

$$
f_{k}\left(t y_{1}+(1-t) y_{2}\right) \leq t f_{k}\left(y_{1}\right)+(1-t) f_{k}\left(y_{2}\right) \leq \bar{d}_{k}^{2} .
$$

Therefore, each $f_{k}(x) \leq \bar{d}_{k}^{2}$ is a convex constraint []. It can be shown that the constraints $\underline{d}_{k}^{2} \leq f_{k}(x)$ are not convex. Thus the constraints in Problem 2 can be classified into two types based on the convexity,

$$
\begin{array}{ll}
\text { Convex constraints: } & f_{k}(x) \leq \bar{d}_{k}^{2}, \\
\text { Non-convex constraints: } & \underline{d}_{k}^{2} \leq f_{k}(x) .
\end{array}
$$

This work is focused on solving the network localization problem keeping the non-convex distance constraints unaltered. Though Doherty, et al. [12 formulated the localization problem as non-convex optimization problem [12] they exclude the non-convex distance constraints to solve the problem using semi-definite programming (SDP). Biswas, et al. 8] converted the non-convex network localization problem into a convex optimization problem by relaxing the non-convex inequality constraints and solved the relaxed problem [13, 14] using SDP [30. A reason behind using SDP method is that the SDP is approximately solvable in polynomial time [5]. Yet none of these approaches solved the general network localization problem.

In this paper, using the Lagrangian theory, the anchor free network localization problem with noisy distance measurements is converted into a root finding problem without any modification of the nonlinear non-convex distance constraints. We solve the root finding problem using an iterative method and prove the convergence of the method to a solution of the localization problem. The method gives an estimation for node positions up to a desired level of accuracy within a real time period. 


\section{Root finding problem construction using Lagrangian func- tion}

The network localization problem is inherently a non-convex optimization problem. In this section, we describe the Lagrangian function with the help of which we transform the general localization problem into a root finding problem. In Problem 2 each non-convex constraint $\underline{d}_{k}^{2} \leq f_{k}(x)$ can be written as $f_{n_{0}+k}(x) \leq \underline{d}_{k}^{2}$ where, $2 \underline{d}_{k}^{2}-f_{k}(x)=f_{n_{0}+k}(x)$. These modifications convert Problem 2 into a non-convex optimization problem as described in Problem 3 .

Problem 3. Given the matrices $\underline{D}=\left(\underline{d}_{i j}\right)_{n \times n}, \bar{D}=\left(\bar{d}_{i j}\right)_{n \times n}$ of the network $\aleph$ consisting of $n$ nodes with unknown positions $x=\left(x_{1}, x_{2}, \cdots, x_{n}\right)$, solve the nonlinear optimization problem:

$\begin{aligned} & \text { Minimize } f_{0}(x) \\ & \text { such that } \quad f_{k}(x) \leq c_{k}, \quad 1 \leq k \leq 2 n_{0}=r(\text { say })\end{aligned}$
where, $c_{k}=\bar{d}_{k}^{2}, 1 \leq k \leq n_{0}$ and $c_{k}=\underline{d}_{k-n_{0}}^{2}, n_{0}+1 \leq k \leq r$.

\subsection{Lagrangian function}

Let $c=\left[c_{0}, c_{1}, c_{2}, \cdots, c_{r}\right]$, where $c_{0}$ is a positive real number independent of $x$ and $c_{k}(1 \leq k \leq r)$ are defined in Problem 3. Note that, $c_{k}>0 \forall k$, because the distance information for each pair of nodes is collected from a network where no two sensors are in the same position.

Definition 1. The Lagrangian function for Problem 3 is defined as,

$$
\mathbb{L}\left(x, c_{0}\right)=\max _{0 \leq k \leq r} \frac{f_{k}(x)}{c_{k}} .
$$

Lagrangian function may be defined in many ways [8] for an optimization problem among which we consider the above form for the Lagrangian function in this paper. Shortly we prove that the Lagrangian function always attains its infimum within the field of interest. With the help of $\mathbb{L}\left(x, c_{0}\right)$ the problem defined in Problem 4 is later proved to be equivalent to 3 under certain restrictions which are acceptable in any real situations.

Problem 4. Let the function $\mathbb{L}\left(x, c_{0}\right)$ attains its infimum at some point $z$ over the domain of definition, i.e.,

$$
\mathbb{L}\left(z, c_{0}\right)=\inf _{x \in \mathbb{R}^{d n}} \max _{0 \leq k \leq r} \frac{f_{k}(x)}{c_{k}} .
$$

We have to find the $z$.

Below we describe a result from [15] which says that, $x$ is an optimal solution of Problem 3 if and only if it is a solution of Problem 4. Thus if we can find a solution $x$ of Problem 4 then $x$ may easily be mapped to an optimal solution of Problem 3 using this result. It may also be noted that this technique does not need the convexity of the constraint functions, i.e. we do not ignore the non-convex constraints from the general problem.

Result 1 ([15]). Let $\bar{x} \in \mathbb{R}^{d n}$ be an optimal solution of the network localization problem as defined by Problem 3 and $c_{0}=f_{0}(\bar{x})>0$. A different $x_{0} \in \mathbb{R}^{d n}$ is an optimal solution of Problem 3 if and only if $x_{0}$ is a solution of the unconstrained problem defined in Problem 4.

In the rest of this section, using Lagrangian theory for non-convex optimization problem [15], we convert Problem 3 into a root finding problem involving single variable. 


\subsection{Lagrange's optimization problem}

The network models under consideration are picked up from networks already embedded in the field of interest. For such an already embedded network, Problem 3 satisfies the following conditions:

1. The problem always has at least one feasible solution, since the graph underlying the network is constructed from a network already embedded in the field of interest.

2. In Problem [3, $\lim _{\|x\| \rightarrow \infty} f_{0}(x)=\infty$. Since in practical applications, the field of interest is always bounded, the feasible region of Problem 3 is also bounded. Since the objective function $f_{0}(x)=\sum_{i=1}^{n}\left\|x_{i}\right\|^{2}$ is everywhere continuous, there always exists some real constant $M$ such that $0<f_{0}(x) \leq M$ for all feasible $x$.

3. Since $f_{0}(x) \leq M$ in the feasible region and Problem 3 has feasible solution,

Problem 3 always possesses an optimal solution, say $\bar{x}$, in $\mathbb{R}^{d n}$. At $x=\bar{x}, f_{0}(\bar{x}) \geq 0$. It may be noted that $f_{0}(\bar{x})=0$ only when all the points are at origin.

4. In Problem 3 the feasible region $X_{0}=\left\{x \in \mathbb{R}^{d n} / f_{k}(x) \leq c_{k}, 1 \leq k \leq r\right\}$ is compact. It is shortly proved in Lemma 1 .

5. Since $f_{0}$ is polynomial, it is uniformly continuous on the feasible region $X_{0}$.

6. For $c_{0} \geq f_{0}(\bar{x})$, there always exists some $x \in \mathbb{R}^{d n}$ such that $f_{0}(\bar{x}) \leq f_{0}(x) \leq c_{0}$ since $f_{0}$ is continuous.

Under the above assumptions, we develop the following result which is used for developing the proposed localization problem.

Lemma 1. In Problem 3 the feasible region

$$
X_{0}=\left\{x=\left(x_{1}, x_{2}, \ldots, x_{n}\right) \in \mathbb{R}^{d n} \mid f_{k}(x) \leq c_{k}, 1 \leq k \leq r\right\}
$$

is compact.

Proof. Without lose of generality we restrict $X_{0}$ in $\mathbb{R}_{+}^{d n}$. Otherwise the origin may be shifted so that the feasible region is included in $\mathbb{R}_{+}^{d n}$.

A set is compact in $\mathbb{R}^{d n}$ if and only if it is both bounded and closed [4. We give an explicit proof of the compactness by showing that the above defined set is both bounded and closed in $\mathbb{R}^{d n}$. In view of the condition (2), $X_{0}$ is bounded.

Closed-ness of $X_{0}$ : Let for an arbitrarily chosen $k, X_{k}=\left\{x \in \mathbb{R}^{d n} / f_{k}(x) \leq c_{k}\right\}$ and $\left\{y^{l}\right\}_{l}$ [where $\left.y^{l}=\left(y_{1}^{l}, y_{2}^{l}, \ldots, y_{n}^{l}\right)\right]$ be a Cauchy sequence in $X_{k}$ with limit $x$. Let $k$-th edge of the grounded graph joins the nodes $i, j$ of the network. For $1 \leq k \leq n_{0}, f_{k}\left(y^{l}\right) \leq c_{k} \Rightarrow\left\|y_{i}^{l}-y_{j}^{l}\right\|^{2} \leq c_{k}$ and if $n_{0}+1 \leq k \leq 2 n_{0}, f_{k}\left(y^{l}\right) \leq c_{k} \Rightarrow 2 c_{k}-\left\|y_{i}^{l}-y_{j}^{l}\right\|^{2} \leq c_{k}$. Let us first consider the case $1 \leq k \leq n_{0}$.

Since $y^{l} \rightarrow x$ therefore for given any $\varepsilon>0$ there exists some $m \in \mathbb{N}$ where for all $l \geq m$

$$
\left\|y^{l}-x\right\|<\varepsilon
$$

This gives for all $l \geq m$,

$$
\left\|y_{u}^{l}-x_{u}\right\|<\varepsilon \quad \text { for each } 1 \leq u \leq n .
$$

Thus for $l \geq m$,

$$
\begin{aligned}
& \left\|x_{i}-x_{j}\right\| \\
= & \left\|x_{i}-y_{i}^{l}+y_{i}^{l}-y_{j}^{l}+y_{j}^{l}-x_{j}\right\| \\
\leq & \left\|x_{i}-y_{i}^{l}\right\|+\left\|y_{i}^{l}-y_{j}^{l}\right\|+\left\|y_{j}^{l}-x_{j}\right\| \\
< & 2 \varepsilon+\left|\sqrt{c_{k}}\right| .
\end{aligned}
$$


Since the above relation holds for arbitrarily chosen $\varepsilon$ we get $\left\|x_{i}-x_{j}\right\| \leq\left|\sqrt{c}_{k}\right|$, i.e., $\left\|x_{i}-x_{j}\right\|^{2} \leq$ $c_{k}$. Thus if $f_{k}\left(y^{l}\right)=\left\|y_{i}^{l}-y_{j}^{l}\right\|^{2}$ then $X_{k}$ is a compact set. For $n_{0}+1 \leq k \leq 2 n_{0}$, the proof for closed-ness is similar as before. Hence for each $k, X_{k}$ is closed. $X_{0}$ is the intersection of finite number of closed sets and it is closed. Therefore $X_{0}$ is compact.

The optimum solution of $\inf _{x \in \mathbb{R}^{d n}} \max _{0 \leq k \leq r} f_{k}(x) / c_{k}$ varies for different values of $c_{0}$ (for $1 \leq k \leq r$, each $c_{k}$ is given in the problem as constants). We define a scalar function $\psi$ of parameter $c_{0} \in \mathbb{R}_{+}-\{0\}$ as follows,

$$
\psi\left(c_{0}\right)=\inf _{x \in \mathbb{R}^{d n}} \max _{0 \leq k \leq r}\left\{f_{k}(x) / c_{k}\right\} .
$$

To construct the Lagrangian optimization problem we here present some results from [15] involving function $\psi\left(c_{0}\right)$.

Result 2 ([15]). Let $\left\{f_{k}:(0 \leq k \leq r)\right\}$ be a finite set of continuously differentiable functions defined on an unbounded set $X, c_{k}>0$. Consider the optimization problem

$$
\begin{aligned}
& \min f_{0}(x) \\
& \text { subject to } f_{k}(x) \leq c_{k}(1 \leq k \leq r)
\end{aligned}
$$

under the following assumptions:

1. The feasible region is compact.

2. $\lim _{\|x\| \rightarrow \infty} f_{0}(x)=\infty$.

3. If $\bar{x}$ is an optimal solution and for any arbitrary constant $c_{0}>f_{0}(\bar{x})$

Then there exists some $x \in X$ such that $f_{0}(\bar{x}) \leq f_{0}(x)<c_{0}$. Also the following conditions hold.

1. $c_{0}<f_{0}(\bar{x}) \Rightarrow \psi\left(c_{0}\right)>1$.

2. With addition to the above conditions if $f_{0}(x)$ is uniformly continuous then $c_{0} \geq f_{0}(\bar{x}) \Rightarrow$ $\psi\left(c_{0}\right) \leq 1$.

3. $\psi$ is a monotone non-increasing continuous function.

4. $c_{0}=f_{0}(\bar{x})$ if and only if $\psi\left(c_{0}\right)=1$.

Theorem 1. Let $\bar{x}$ be an optimal solution of Problem 3 , Then $\psi\left(c_{0}\right)$ has the following properties:

1. If $c_{0}<f_{0}(\bar{x})$ then $\psi\left(c_{0}\right)>1$.

2. If $c_{0} \geq f_{0}(\bar{x})$ then $\psi\left(c_{0}\right) \leq 1$.

3. $\psi$ is a non-increasing continuous function of $c_{0}$.

4. $c_{0}=f_{0}(\bar{x})$ if and only if $\psi\left(c_{0}\right)=1$.

Proof. Under the network model, we have seen that $f_{i}$ s in Problem 3 are continuously differentiable and $c_{i}>0$. The above mentioned condition (4) of the underlying network model shows that the feasible region of Problem 3 is compact. The condition (3) shows that the Problem 3 has an optimal solution, say $\bar{x}$. By the condition (6), there always exists some $x \in \mathbb{R}^{d n}$ such that $f_{0}(\bar{x}) \leq f_{0}(x) \leq c_{0}$ when $c_{0} \geq f_{0}(\bar{x})$. The proof of this theorem then follows from Problem 2.

In the following paragraph, we present the network localization problem as a root finding problem which may be obtained from Problem 2 using Theorem 1 .

Problem 5. Given the matrices $\underline{D}=\left(\underline{d}_{i j}\right)_{n \times n}, \bar{D}=\left(\bar{d}_{i j}\right)_{n \times n}$ of the network $\aleph$ with a set $X$ of $n$ nodes with unknown positions. Let $\psi\left(c_{0}\right)=\inf _{x \in \mathbb{R}^{d n}} \max _{0 \leq k \leq r}\left\{f_{k}(x) / c_{k}\right\}$.

Find $c_{0}$ such that $\psi\left(c_{0}\right)=1$.

To get a good estimation for the node positions in the network, we need to search for some positive real number $c$ for which there exists some $x \in \mathbb{R}^{d n}$ such that the value of the function $\psi$ is equal or very close to 1 . In the rest of this paper, we will concentrate for finding or estimating the roots of $\psi\left(c_{0}\right)=1$. 


\section{Solving the root finding problem}

In the previous section, we have seen that solving the network localization problem is equivalent to solving Problem 5. Here we prove that if we can find a root $c_{0}=c_{0}^{*}$ of the equation $\psi\left(c_{0}\right)=1$, node positions of the network will be obtained from the corresponding $x$ at which $\mathbb{L}\left(x, c_{0}\right)(=$ $\left.\max _{0 \leq k \leq r}\left\{f_{k}(x) / c_{k}\right\}\right)$ exactly equals 1 .

Theorem 2. Let $c_{0}^{*}$ is a root of the equation $\psi\left(c_{0}\right)=1$. Then there exists an optimizing $x=x^{*}$ such that,

$$
\psi\left(c_{0}^{*}\right)=\mathbb{L}\left(x^{*}, c_{0}^{*}\right)=1 .
$$

Proof. Since the feasible region of the network localization problem is compact the optimal solution of the problem lies within a compact set. Therefore instead of searching the minimizing $x$ of the function $\max _{0 \leq k \leq r, c_{0}=c_{0}^{*}}\left\{f_{k}(x) / c_{k}\right\}$ all over $\mathbb{R}^{d n}$ we may restrict our search on a compact subset, say $K \subset \mathbb{R}^{n d}$, containing the feasible region of the network localization problem. Such a compact set for Problem 5 may be constructed as follows:

Consider the field of interest, $F$, for localizing the network in $\mathbb{R}^{d}$. If $x$ is a feasible solution of the general network localization problem somewhere in $\mathbb{R}^{d}$ then by using the translation and rotation operations we may get a congruent realization of the network in $F$. Since $F$ is bounded we will get some upper bound as well as lower bound for each coordinate of any point lying in the region. If we get any localization of the network obtained by solving Problem 5 then it will lie within the field of interest.

Let $M^{\prime}$ and $m^{\prime}$ be the maximum and minimum for all of the $d$ coordinates in the field of interest. Consider a $d n$-dimensional box

$$
K=\left\{\left(y_{1}, y_{2}, \ldots, y_{i}, \ldots, y_{d n}\right) \mid m^{\prime} \leq y_{i} \leq M^{\prime}, \forall i\right\}
$$

in $\mathbb{R}^{d n}$. $K$ is always compact. Let $x=\left(x_{1}, \ldots, x_{n}\right)$ be a realization of the network within the field of interest. For each $i$, if $x_{i}=\left(x_{i 1}, x_{i 2}, \ldots, x_{i d}\right)$ then $m^{\prime} \leq x_{i j} \leq M^{\prime}$. Therefore corresponding to each solution of the network localization problem there is a point in the $d n$-dimensional box.

The function $\mathbb{L}\left(x, c_{0}^{*}\right)$ is a continuous function of the variable $x$ on this compact set $K$. The proof of the theorem will be followed if we can show that the continuous function $\mathbb{L}\left(x, c_{0}^{*}\right)$ defined on $K$ attains its minimum at some point in $K$.

Since $K$ is a compact set and the function $\mathbb{L}$ is continuous, $\mathbb{L}\left(K, c_{0}^{*}\right)$ is a compact set (i.e., closed and bounded). Also the infimum of any set is either a limit point or an element of the set. In both cases, the infimum of $\mathbb{L}\left(K, c_{0}^{*}\right)$ lies inside $\mathbb{L}\left(K, c_{0}^{*}\right)$ since, $\mathbb{L}\left(K, c_{0}^{*}\right)$ is closed. Therefore, we get some $x^{*}$ such that $\mathbb{L}\left(x^{*}, c_{0}^{*}\right)=\inf _{x \in K} \mathbb{L}\left(x, c_{0}^{*}\right)$ i.e., $\psi\left(c_{0}^{*}\right)=\mathbb{L}\left(x^{*}, c_{0}^{*}\right)=1$.

We develop an iterative method in light of the bisection method for finding a root of $\psi\left(c_{0}\right)=1$, $\left(c_{0} \in \mathbb{R}\right)$. The method is guaranteed to converge to a root of the continuous function $\psi\left(c_{0}\right)-1$ on an interval $\left[c_{01}, c_{02}\right]$, if $\left(\psi\left(c_{01}\right)-1\right)$ and $\left(\psi\left(c_{02}\right)-1\right)$ have opposite signs. At the initial stage of the iterative method we search for an interval containing $c_{0}$ within which $\psi\left(c_{0}\right)-1$ must have a root. The searching process may progress as follows:

Consider a real number $c_{0}=c_{01}>0$. For computing $\psi\left(c_{01}\right)-1$ it is required to solve a finite minimax problem which is an NP-hard problem [11. We use smoothing gradient technique (Section 4.1) for computing an approximate value of $\psi\left(c_{01}\right)-1$. In this smoothing technique, $\psi\left(c_{01}\right)-1$ may be approximated such that depending on the approximated value of the function we will get an interval within which the actual functional value lies. Using these bounds and the monotonic non-increasing property of the function we determine the sign of $\psi\left(c_{01}\right)-1$ (Theorem 3). If $c_{01}$ is not a root of $\psi\left(c_{0}\right)-1$ then one of the following cases may occur.

case $1 .\left(\psi\left(c_{01}\right)-1\right.$ is positive): Choose a point $c_{0}=c_{02}\left(=c_{01}+\alpha, \alpha\right.$ is an arbitrary positive number). Since $\psi\left(c_{0}\right)-1$ is a non-increasing continuous function of $c_{0}$ (Theorem 1), then for sufficiently large constant $\alpha$ either $\psi\left(c_{02}\right)-1=0$ or $\psi\left(c_{02}\right)-1<0$. If $\psi\left(c_{02}\right)-1=0$ then $c_{02}$ is a root of the equation $\psi\left(c_{0}\right)=1$ and we are done. Otherwise the required interval is $\left[c_{01}, c_{02}\right]$. 
case 2. $\left(\psi\left(c_{01}\right)-1\right.$ is negative at $\left.c_{01}\right)$ : Choose a point $c_{0}=c_{02}\left(0<c_{02}<c_{01}\right)$. With similar reason as in case 1 , either $\psi\left(c_{02}\right)-1=0$ or $\psi\left(c_{02}\right)-1>0$. If $\psi\left(c_{02}\right)-1=0$ then we are done, otherwise the required interval is $\left[c_{02}, c_{01}\right]$.

In this way without computing the exact value of $\psi\left(c_{0}\right)-1$ we obtain an interval $\left[c_{01}, c_{02}\right]$ at the end points of which $\psi\left(c_{0}\right)-1$ take values with opposite signs and proceed for finding a solution of $\psi\left(c_{0}\right)-1$. In the following section we describe the smoothing gradient technique which we implemented for approximately computing $\psi\left(c_{0}\right)-1$.

\subsection{Smoothing Gradient Technique for solving finite mini-max}

In the literature there are several smoothing techniques which may be used for solving a finite minimax problem. Among those techniques we choose one for solving our finite mini-max optimization problem in which the function $\psi\left(c_{0}\right)-1$ remains bounded for each $c_{0} \in \mathbb{R}$. The technique uses a smoothing function (given in (4)) to approximate the underlying non-smooth objective function $L\left(x, c_{0}\right)$. A smoothing function for a given non-smooth continuous function may be defined as follows.

Definition 2. [22] Let, $f: \mathbb{R}^{n} \rightarrow \mathbb{R}$ be a continuous non-smooth function. We call $\tilde{f}: \mathbb{R}^{n} \times \mathbb{R}_{+} \rightarrow \mathbb{R}^{2}$ a smoothing function of $f$ if $\tilde{f}(., \mu)$ is continuously differntiable in $\mathbb{R}^{n}$ for every $\mu \in \mathbb{R}^{+}$and

$$
\lim _{z \rightarrow x, \mu \downarrow 0} \tilde{f}(z, \mu)=f(x)
$$

for any $x \in \mathbb{R}^{n}$.

To solve the root finding problem we require an estimation for $\min _{x} L\left(x, c_{0}\right)=\min _{x} \max _{k}\left\{\frac{f_{k}(x)}{c_{k}}\right\}$ $\left(c_{0} \in\left[c_{01}, c_{02}\right]\right)$, where $L\left(x, c_{0}\right)$ is a non-smooth continuous function. We consider the smoothing function for $\mathbb{L}\left(x, c_{0}\right)$ as follows:

$$
\tilde{\mathbb{L}}\left(x, \mu, c_{0}\right)=\mu \log \sum_{k=1}^{r} \exp \left\{\frac{1}{\mu} \times \frac{f_{k}(x)}{c_{k}}\right\} .
$$

The function $\tilde{\mathbb{L}}\left(x, \mu, c_{0}\right)$ provides a good estimation for $\mathbb{L}\left(x, c_{0}\right)$ since the following inequality holds.

Theorem 3. $\mathbb{L}\left(x, c_{0}\right) \leq \tilde{\mathbb{L}}\left(x, \mu, c_{0}\right) \leq \mathbb{L}\left(x, c_{0}\right)+\mu \log r$, for $\mu>0$.

Proof.

$$
\begin{aligned}
\tilde{\mathbb{L}}\left(x, \mu, c_{0}\right)-\mathbb{L}\left(x, c_{0}\right) & =\mu \log \sum_{k=1}^{r} \exp \left\{\frac{1}{\mu} \times \frac{f_{k}(x)}{c_{k}}\right\}-\mu \times\left(\frac{1}{\mu} \times \mathbb{L}\left(x, c_{0}\right)\right) \\
& =\mu \log \sum_{k=1}^{r} \exp \left\{\frac{1}{\mu} \times \frac{f_{k}(x)}{c_{k}}\right\}-\mu \log \exp \left(\frac{1}{\mu} \times \mathbb{L}\left(x, c_{0}\right)\right) \\
& =\mu \log \left\{\sum_{k=1}^{r} \exp \left\{\frac{1}{\mu} \times \frac{f_{k}(x)}{c_{k}}\right\} / \exp \left(\frac{1}{\mu} \times \mathbb{L}\left(x, c_{0}\right)\right)\right\} \\
& =\mu \log \left\{\sum_{k=1}^{r} \exp \left\{\frac{1}{\mu} \times \frac{f_{k}(x)}{c_{k}}-\frac{1}{\mu} \times \mathbb{L}\left(x, c_{0}\right)\right\}\right\} \\
& =\mu \log \left\{\sum_{k=1}^{r} \exp \left(\frac{1}{\mu} \times\left\{\frac{f_{k}(x)}{c_{k}}-\mathbb{L}\left(x, c_{0}\right)\right\}\right)\right\}
\end{aligned}
$$

Since $\frac{f_{k}(x)}{c_{k}} \leq \mathbb{L}\left(x, c_{0}\right)=\max _{0 \leq k \leq r} \frac{f_{k}(x)}{c_{k}}$, at any point $x \in \mathbb{R}^{n d}$,

$$
\exp \left(\frac{1}{\mu} \times\left\{\frac{f_{k}(x)}{c_{k}}-\mathbb{L}\left(x, c_{0}\right)\right\}\right) \leq 1
$$


for each $k$. This gives,

$$
\sum_{k=0}^{r} \exp \left(\frac{1}{\mu} \times\left\{\frac{f_{k}(x)}{c_{k}}-\mathbb{L}\left(x, c_{0}\right)\right\}\right) \leq r
$$

Hence,

$$
\tilde{\mathbb{L}}\left(x, \mu, c_{0}\right)-\mathbb{L}\left(x, c_{0}\right) \leq \mu \log r \Rightarrow \tilde{\mathbb{L}}\left(x, \mu, c_{0}\right) \leq \mathbb{L}\left(x, c_{0}\right)+\mu \log r .
$$

Also for each $x \in \mathbb{R}^{n d}$ there is some $k=m$ for which

$$
\frac{f_{m}(x)}{c_{m}}=\mathbb{L}\left(x, c_{0}\right)=\max _{0 \leq k \leq r} \frac{f_{k}(x)}{c_{k}} .
$$

Thus we get,

$$
\exp \left(\frac{1}{\mu} \times\left\{\frac{f_{m}(x)}{c_{m}}-\mathbb{L}\left(x, c_{0}\right)\right\}\right)=1
$$

which gives

$$
\sum_{k=0}^{r} \exp \left(\frac{1}{\mu} \times\left\{\frac{f_{k}(x)}{c_{k}}-\mathbb{L}\left(x, c_{0}\right)\right\}\right) \geq 1
$$

or,

$$
\mu \log \left(\sum_{k=0}^{r} \exp \left(\frac{1}{\mu} \times\left\{\frac{f_{k}(x)}{c_{k}}-\mathbb{L}\left(x, c_{0}\right)\right\}\right)\right) \geq 0
$$

i.e.,

$$
\tilde{\mathbb{L}}\left(x, \mu, c_{0}\right) \geq \mathbb{L}\left(x, c_{0}\right) .
$$

Combining Equation 5 and Equation 6 we get the given inequality.

We describe the smoothing gradient algorithm below which will produce a clarkr stationary point (Appendix A) $x_{0}$ for $\min _{x} L\left(x, c_{0}\right)$.

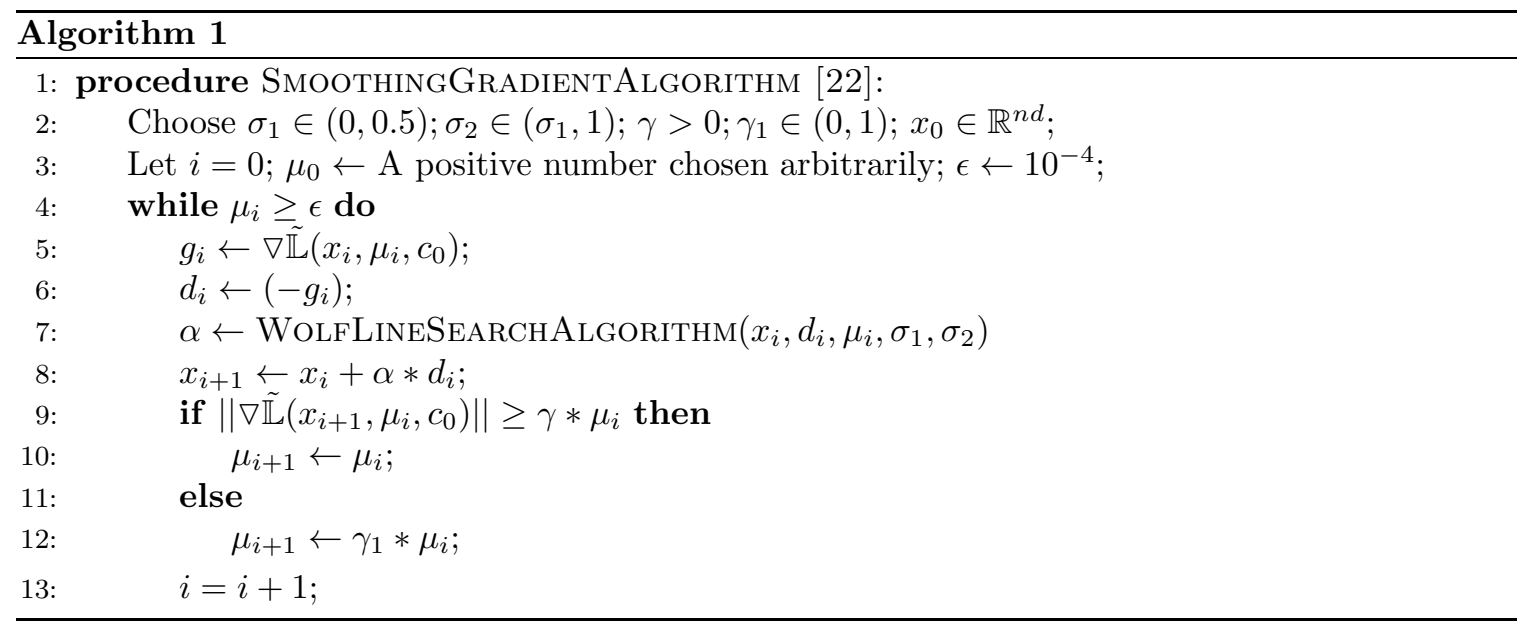

In each step of the Smoothing Gradient Algorithm we use WolfLineSearchAlgoRITHM [1] for finding $\alpha$ for the next iteration. The algorithm searches for finding the maximum value of the constant $\alpha$ satisfying the following two conditions.

1. $\tilde{\mathbb{L}}\left(x_{i}+\alpha d_{i}, \mu_{i}, c_{0}\right) \leq \tilde{\mathbb{L}}\left(x_{i}, \mu_{i}, c_{0}\right)+\sigma_{1} \alpha g_{i}^{T} d_{i}$

2. $\nabla \tilde{\mathbb{L}}\left(x_{i}+\alpha d_{i}, \mu_{i}, c_{0}\right)^{T} d_{i} \geq \sigma_{2} g_{i}^{T} d_{i}$ 
where $x_{i}, d_{i}, \mu_{i}, \sigma_{1}, \sigma_{2}$ are from the smoothing gradient algorithm. The first condition ensures that at the $i+1$-th step of the iteration the functional value $\tilde{\mathbb{L}}\left(x_{i}+\alpha d_{i}, \mu_{i}, c_{0}\right)$ is smaller than $\tilde{\mathbb{L}}\left(x_{i}, \mu_{i}, c_{0}\right)$ (since $\sigma_{1} \alpha g_{i}^{T} d_{i}=-\sigma_{1} \alpha\left\|g_{i}\right\|^{2}$ is negative). Here we show that an $\underset{\sim}{\alpha}$ satisfying this condition always exists. From the Taylor theorem for multivariate functions [4] of $\tilde{\mathbb{L}}\left(x_{i}+\alpha d_{i}, \mu_{i}, c_{0}\right)$ we get

$$
\tilde{\mathbb{L}}\left(x_{i}+\alpha d_{i}, \mu_{i}, c_{0}\right)=\tilde{\mathbb{L}}\left(x_{i}, \mu_{i}, c_{0}\right)+\alpha g_{i}^{T} d_{i}+O\left(\alpha^{2}\right)[22]
$$

Therefore

$$
\begin{array}{ll} 
& \tilde{\mathbb{L}}\left(x_{i}, \mu_{i}, c_{0}\right)+\alpha g_{i}^{T} d_{i}+O\left(\alpha^{2}\right) \leq \tilde{\mathbb{L}}\left(x_{i}, \mu_{i}, c_{0}\right)+\sigma_{1} \alpha g_{i}^{T} d_{i} \\
\text { if, } & \alpha g_{i}^{T} d_{i}+O\left(\alpha^{2}\right) \leq \sigma_{1} \alpha g_{i}^{T} d_{i} \\
\text { i.e. if, } & -\left(1-\sigma_{1}\right) \alpha\left\|g_{i}\right\|^{2}+O\left(\alpha^{2}\right) \leq 0, \text { or },\left(1-\sigma_{1}\right) \alpha\left\|g_{i}\right\|^{2} \geq O\left(\alpha^{2}\right) .
\end{array}
$$

Using the Taylor's theorem it may be concluded that such an $\alpha$ always exists. Condition (2) of WolfLineSEARChAlgorithm has been inserted to keep $\alpha$ sufficiently large as such the slope of $\tilde{\mathbb{L}}\left(x_{i}+\alpha d_{i}, \mu_{i}, c_{0}\right)$ remains at least $\sigma_{2}\left(\sigma_{1}<\sigma_{2}<1\right)$ times larger than the slope of $\tilde{\mathbb{L}}\left(x_{i}, \mu_{i}, c_{0}\right)$ [1].

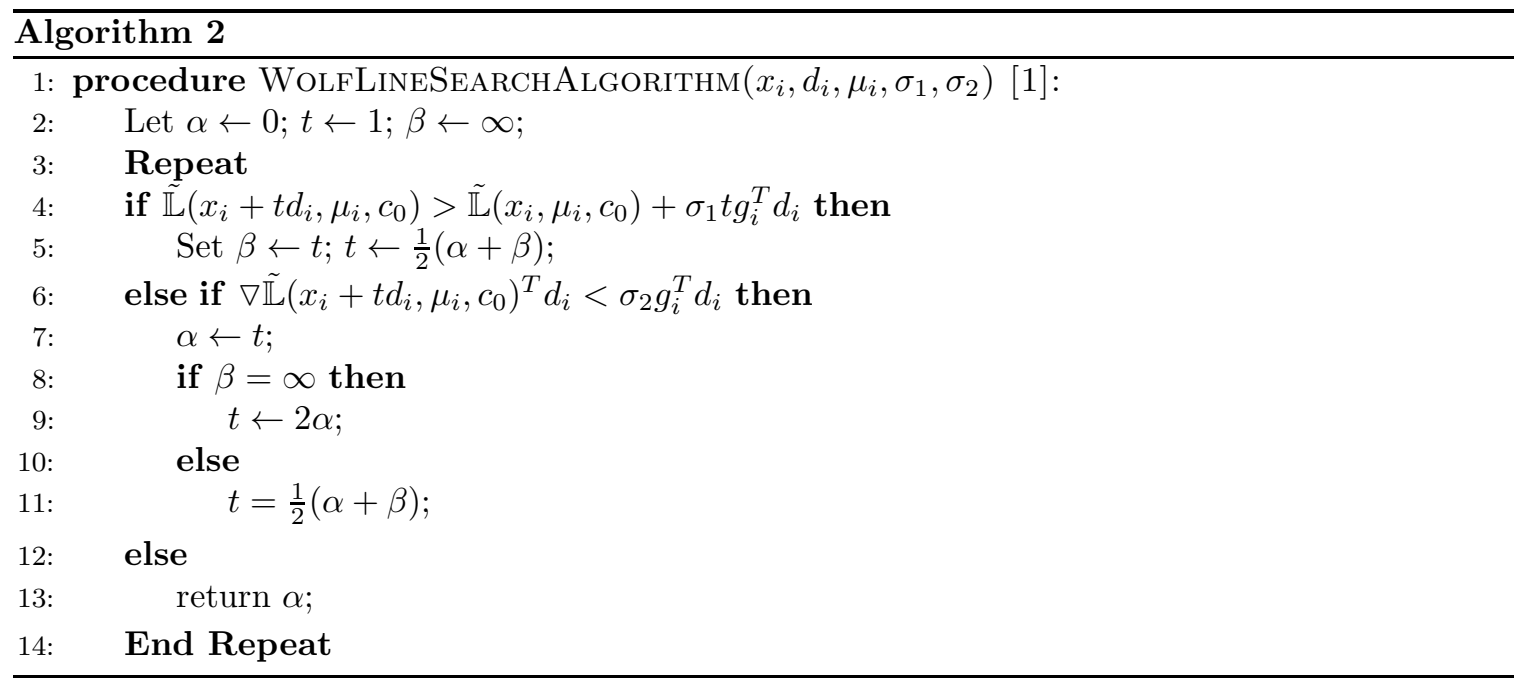

\section{Convergence analysis of the root finding method}

The convergence of the root finding method inherently depends on the convergence of the SMOOTHINGGRADIENTALGORITHM.

\subsection{Convergence of SmoothingGradientAlgorithm}

Let $\left\{x_{i}\right\}$ and $\left\{\mu_{i}\right\}$ are the sequences generated by the smoothing gradient algorithm. Towards proving the convergence of the method let us first consider the set

$$
S=\left\{i \mid \mu_{i+1}=\gamma_{1} \mu_{i}\right\}
$$

in the smoothing gradient algorithm.

Lemma 2. The set $S$ can not be finite.

Proof. If $S$ is a finite set then from the smoothing gradient algorithm we get, there exists an integer $i^{\prime}$ such that for all $i>i^{\prime}$,

$$
\left\|\nabla \tilde{\mathbb{L}}\left(x_{i+1}, \mu_{i}, c_{0}\right)\right\| \geq \gamma * \mu_{i},
$$

and $\mu_{i}=\mu_{i^{\prime}}=\mu$ (say). If this is true then our claim is that,

$$
\liminf _{i \rightarrow \infty}\left\|\nabla \tilde{\mathbb{L}}\left(x_{i+1}, \mu, c_{0}\right)\right\|=0 .
$$


Suppose that (8) does not hold. Then there exists a sub-sequence of $\left\{g_{i}\right\}\left(g_{i}=\nabla \tilde{\mathbb{L}}\left(x_{i+1}, \mu, c_{0}\right)\right)$, say $\left\{g_{j}\right\}$, for which

$$
\left\|g_{j}\right\| \geq \epsilon \text { for some } \epsilon>0 \text { and } \forall j .
$$

But in Wolfe Line Search Algorithm $d_{i}\left(=-g_{i}\right)$ is always a decent direction for the gradient [1]. Thus the sequence $\left\{\tilde{\mathbb{L}}\left(x_{j}, \mu, c_{0}\right)\right\}$ generated by the algorithm is a monotonically decreasing sequence. Also $x_{j} \in K(\forall j)$ where $K$ is the compact set (we have chosen in our paper). Therefore the sequence $\left\{\tilde{\mathbb{L}}\left(x_{j}, \mu, c_{0}\right)\right\}$ will be bounded below and convergent in $K$. Hence we get,

$$
\tilde{\mathbb{L}}\left(x_{j}, \mu, c_{0}\right)-\tilde{\mathbb{L}}\left(x_{j+1}, \mu, c_{0}\right) \rightarrow 0 \text {, as } j \rightarrow \infty .
$$

Using this and the condition (1) of wolfe line search we obtain,

$$
-g_{j}^{T} \alpha_{j} d_{j} \leq \frac{1}{\sigma_{1}}\left(\tilde{\mathbb{L}}\left(x_{j}, \mu, c_{0}\right)-\tilde{\mathbb{L}}\left(x_{j+1}, \mu, c_{0}\right)\right) \rightarrow 0 \quad \text { as } j \rightarrow \infty .
$$

This gives $-g_{j}^{T} \alpha_{j} d_{j} \rightarrow 0$ as $j \rightarrow \infty$. But $-g_{j}^{T} \alpha_{j} d_{j}=\left\|g_{j}\right\| .\left\|\alpha_{j} d_{j}\right\| \cos \theta$ where $\theta=\pi$ is the angle in between $g_{j}$ and $d_{j}$. Thus we get

$$
\left\|\alpha_{j} d_{j}\right\| \rightarrow 0 \text { since }\left\|g_{j}\right\| \geq \epsilon
$$

Since $x_{j+1}=x_{j}+\alpha_{j} d_{j}$ and $\nabla \tilde{\mathbb{L}}$ differntiable on $K$, the Taylor's Theorem [4] on $\nabla \tilde{\mathbb{L}}\left(x_{j+1}, \mu, c_{0}\right)$ gives that,

$$
\nabla \tilde{\mathbb{L}}\left(x_{j}+\alpha_{j} d_{j}, \mu, c_{0}\right)^{T} \alpha_{j} d_{j}=\nabla \tilde{\mathbb{L}}\left(x_{j}, \mu, c_{0}\right)^{T} \alpha_{j} d_{j}+\alpha_{j} d_{j} \nabla^{2} \tilde{\mathbb{L}}\left(x_{j}, \mu, c_{0}\right)^{T} \alpha_{j} d_{j}+o\left(\left\|\alpha_{j} d_{j}\right\|\right)
$$

or,

$$
\nabla \tilde{\mathbb{L}}\left(x_{j}+\alpha_{j} d_{j}, \mu, c_{0}\right)^{T} \alpha_{j} d_{j}=\nabla \tilde{\mathbb{L}}\left(x_{j}, \mu, c_{0}\right)^{T} \alpha_{j} d_{j}+o\left(\left\|\alpha_{j} d_{j}\right\|\right)
$$

This gives,

$$
\lim _{j \rightarrow \infty} \frac{\nabla \tilde{\mathbb{L}}\left(x_{j+1}, \mu, c_{0}\right)^{T} \alpha_{j} d_{j}}{\nabla \tilde{\mathbb{L}}\left(x_{j}, \mu, c_{0}\right)^{T} \alpha_{j} d_{j}}=1,
$$

since from (10) $o\left(\left\|\alpha_{j} d_{j}\right\|\right) \approx 0$ for $j \rightarrow \infty$. But (11) gives a contradiction since from the second condition of wolfe line search algorithm we get the following

$$
\begin{aligned}
& \nabla \tilde{\mathbb{L}}\left(x_{j+1}, \mu, c_{0}\right)^{T} d_{j} \geq \sigma_{2} g_{j}^{T} d_{j} \\
& \text { or, } \quad-g\left(x_{j+1}\right)^{T} g\left(x_{j}\right) \quad \geq \quad-\sigma_{2} g\left(x_{j}\right)^{T} g\left(x_{j}\right) \\
& \text { or, } \quad g\left(x_{j+1}\right)^{T} g\left(x_{j}\right) \leq \sigma_{2} g\left(x_{j}\right)^{T} g\left(x_{j}\right) \\
& \text { i.e., } \quad \frac{g\left(x_{j+1}\right)^{T} g\left(x_{j}\right)}{g\left(x_{j}\right)^{T} g\left(x_{j}\right)} \leq \sigma_{2}<1 \\
& \text { or, } \quad \frac{\nabla \tilde{\mathbb{L}}\left(x_{j+1}, \mu, c_{0}\right)^{T} \alpha_{j} d_{j}}{\nabla \tilde{\mathbb{L}}\left(x_{j}, \mu, c_{0}\right)^{T} \alpha_{j} d_{j}} \leq \sigma_{2}<1
\end{aligned}
$$

Therefore $\lim _{j \rightarrow \infty} \frac{\nabla \tilde{\mathbb{L}}\left(x_{j+1}, \mu, c_{0}\right)^{T} \alpha_{j} d_{j}}{\nabla \tilde{\mathbb{L}}\left(x_{j}, \mu, c_{0}\right)^{T} \alpha_{j} d_{j}} \neq 1$ and $\left\|g_{j}\right\| \geq \epsilon$ does not hold and we get,

$$
\liminf _{i \rightarrow \infty}\left\|\nabla \tilde{\mathbb{L}}\left(x_{i+1}, \mu, c_{0}\right)\right\|=0 .
$$

But Equation (12) contradicts Equation (7) since $\gamma \mu$ is a constant. Thus $S$ can not be a finite set.

Lemma 3. For the sequence $\left\{x_{i}\right\}$ and $\left\{\mu_{i}\right\}$ generated by the smoothing gradient algorithm the following conditions hold, 
1. $\lim _{i \rightarrow \infty} \mu_{i}=0$.

2. $\lim _{i \rightarrow \infty}\left\|\nabla \tilde{\mathbb{L}}\left(x_{i+1}, \mu_{i}, c_{0}\right)\right\|=0$.

Proof. We use Lemma 2 to prove both these results.

1. Since $S$ is an infinite set $\mu_{i+1}=\mu_{i} \gamma_{1}\left(\gamma_{1}<1\right)$ for infinitely many $i$-s in the smoothing gradient algorithm. Therefore $\lim _{i \rightarrow \infty} \mu_{i}=0$.

2. $\lim _{i \rightarrow \infty}\left\|\nabla \tilde{\mathbb{L}}\left(x_{i+1}, \mu_{i}, c_{0}\right)\right\| \leq \gamma \lim _{i \rightarrow \infty} \mu_{i}=0$.

Since in Lemma 3, $\lim _{i \rightarrow \infty} \mu_{i}=0$ therefore $\lim _{\mu_{i} \downarrow 0} \tilde{\mathbb{L}}\left(x_{i+1}, \mu_{i}, c_{0}\right)=\mathbb{L}\left(x_{i+1}, c_{0}\right)$ at each $x_{i+1}$. The following theorem can be proved by using Lemma 3 .

Theorem 4. [10, 31] Any point $x_{0}$ generated by the smoothing gradient algorithm is a clarkr stationary point of $\mathbb{L}$ at $c_{0} \in \mathbb{R}$.

A clarkr stationary point is a solution of $\min _{x} \mathbb{L}\left(x, c_{0}\right)$ [10. Hence the smoothing gradient algorithm converges to a solution of $\min _{x} \mathbb{L}\left(x, c_{0}\right)$.

\subsection{Convergence of the Root Finding Method:}

Theorem 5. Let $\left\{y_{i}\right\},\left\{\mu_{i}\right\}$ and $\left\{c_{i}\right\}$ be the sequences generated by the root finding iterative method. As $\left\{c_{i}\right\}$ converges to a root $c_{0}^{*}$ of the equation $\psi\left(c_{0}\right)=1$ the following two conditions will hold,

$$
\begin{aligned}
& \text { 1. } \lim _{i \rightarrow \infty} \mu_{i}=0 \text { and } \\
& \text { 2. } \lim _{i \rightarrow \infty}\left\|\nabla \tilde{\mathbb{L}}\left(y_{i+1}, \mu_{i}, c_{0}\right)\right\|=0 \text {. }
\end{aligned}
$$

Proof. We use Lemmma 3 to prove this theorem.

1. Let at the $i$-th step of the root finding iterative method we choose $\mu_{i 0}=\frac{1}{i}$ as initial guess for $\mu_{0}$ to start the smoothing gradient algorithm. At the end of the iteration let we obtain $\mu_{i}$ as the final value of $\mu$. Then $\mu_{i} \leq \mu_{i 0}=\frac{1}{i}$. Hence $\lim _{i \rightarrow \infty} \mu_{i}=0$ follows since $\lim _{i \rightarrow \infty} \mu_{i 0}=0$.

2. From condition (2) of Lemma 3 we get, at the $i$-th step of the root finding method $\| \nabla \tilde{\mathbb{L}}$ $\left(y_{i+1}, \mu_{i}, c_{0}\right) \|$ can be made less than $\frac{1}{i}$ by increasing the number of iteration in the smoothing gradient algorithm. Hence the proof follows similarly as (1).

Using Theorem 4 we immediately get the following theorem.

Theorem 6. As $\left\{c_{i}\right\}$ converges to a root $c_{0}^{*}$ of the equation $\psi\left(c_{0}\right)=1$ the iterative root finding method converges to a clarkr stationary point of the non-smooth function $\mathbb{L}\left(x, c_{0}\right)$. 


\subsection{Some Instances showing the performance of the proposed method}

In our work we consider different networks with noisy distances having noise up to $15 \%$ over the exact distances between adjacent pair of nodes. We choose a $10 \times 10$ square region as the field of interest (we don't specify any unit for this distances since units can be chosen as required in the relevant field). We randomly deploy the networks having within this region and select a random set of vertex pairs as the edge set. The exact distances between adjacent pairs of nodes are measured and recorded. The maximum distance possible between such pairs of nodes is called diameter $(=10 \sqrt{2})$ of the square region. To maintain a $15 \%$ noise in the distance measurement, we choose a random number from $[-10 \sqrt{2} \times 0.15,10 \sqrt{2} \times 0.15] \approx[-2,2]$ corresponding to each edge and add it to its exact distance as error. Under this setting, the estimated positions of nodes for different networks obtained from our algorithm is compared with their original positions.

Example 1. We consider a network consisting of 10 nodes and 28 communication links with weights of the links as the noisy distances. The original and estimated positions of node in the network obtained from our algorithm are recorded in Table 1 .

\begin{tabular}{|c|c|c|}
\hline Vertices & $(x, y)$ (original) & $(x, y)$ (estimated) \\
\hline 1 & $(0,0)$ & $(0,0)$ \\
\hline 2 & $(4.0122,-0.0000)$ & $(4.1359,0.0000)$ \\
\hline 3 & $(3.8810,-2.4025)$ & $(4.0522,-2.3175)$ \\
\hline 4 & $(6.1459,-1.8400)$ & $(6.3297,-1.6593)$ \\
\hline 5 & $(7.9481,-0.3167)$ & $(7.9421,0.1764)$ \\
\hline 6 & $(2.1969,-0.5585)$ & $(2.3947,-0.6566)$ \\
\hline 7 & $(6.1260,5.7528)$ & $(0.0901,2.5287)$ \\
\hline 8 & $(6.8309,6.0573)$ & $(6.6231,6.2059)$ \\
\hline 9 & $(3.9878,4.2560)$ & $(3.7412,4.3045)$ \\
\hline 10 & $(4.2515,2.2306)$ & $(4.0891,2.3111)$ \\
\hline
\end{tabular}

Table 1: The original and estimated node positions of an arbitrary network

The Figure 2(a) and Figure 2(b) represents two different realizations of the network among which Figure 2(a) corresponds to the node positions given in Table 1, The network is not uniquely localizable since the node 7 has degree 2. As shown in Figure 2 the node 7 may be assigned at least two different positions in any particular assignment of positions for the other nodes of the network in the plane. The Figure 3 represents the estimated node positions for the network using root finding method. The estimated node positions of the network (Figure 3) are within small neighborhood of the realization of the network given in Figure Q2(b).

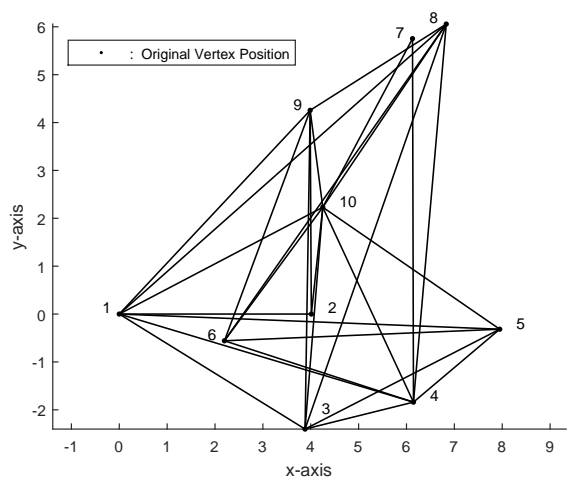

Figure 2: (a)

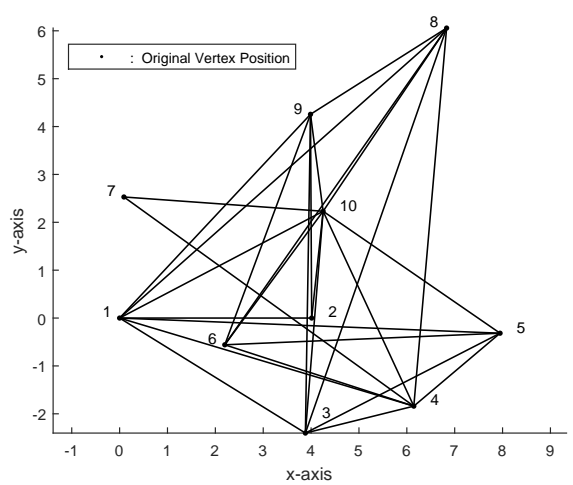

(b) 


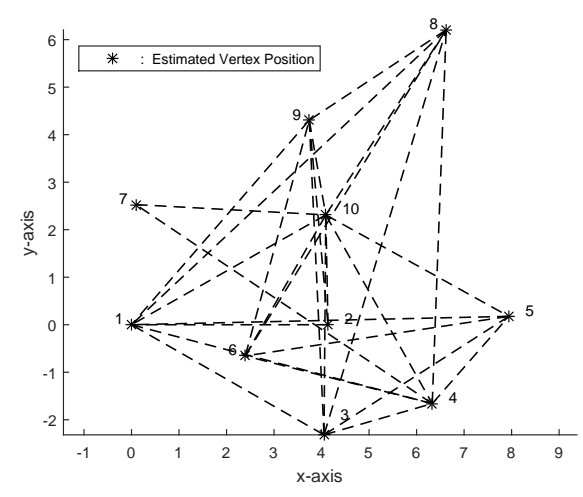

Figure 3: The estimated node positions in the network in Figure 2

If a network is not uniquely localizable the associated localization problem has multiple solutions satisfying the distance constraints. The above measurement leads an intuition that the proposed method gives good estimation for node positions.

Example 2. In Table 0 we are showing the original and estimated node positions for an uniquely localizable network. Figure 4 represents the associated networks along with their communication links.

\begin{tabular}{|c|c|c|}
\hline Vertices & $(x, y)$ (original) & $(x, y)$ (estimated) \\
\hline 1 & $(0,0)$ & $(0,0)$ \\
\hline 2 & $(3.6881,0.0000)$ & $(3.7735,0.0000)$ \\
\hline 3 & $(1.8213,0.2799)$ & $(1.7589,0.3499)$ \\
\hline 4 & $(3.6192,2.4434)$ & $(3.4261,2.5843)$ \\
\hline 5 & $(2.5173,0.8943)$ & $(2.2052,0.1595)$ \\
\hline 6 & $(3.4462,2.4396)$ & $(3.3709,2.2453)$ \\
\hline 7 & $(5.6695,4.4681)$ & $(5.5768,4.5106)$ \\
\hline 8 & $(0.6039,5.6479)$ & $(0.6087,5.6920)$ \\
\hline 9 & $(2.3696,3.3278)$ & $(2.3902,3.3853)$ \\
\hline 10 & $(3.4283,0.1664)$ & $(3.7578,0.0090)$ \\
\hline 11 & $(3.9853,4.5081)$ & $(4.1825,4.3951)$ \\
\hline 12 & $(3.8426,1.3047)$ & $(3.8101,1.2018)$ \\
\hline 13 & $(1.4380,5.3479)$ & $(1.1181,5.7930)$ \\
\hline 14 & $(2.3117,1.9807)$ & $(2.2834,2.0298)$ \\
\hline 15 & $(4.5512,2.6738)$ & $(4.5605,2.7180)$ \\
\hline
\end{tabular}

Table 2: The original and estimated node positions of an uniquely localizable network

We have seen that the estimated node positions are very close to the unique solution of the localization problem.

Observations 1. Either a network is uniquely localizable or not the root finding method gives a good estimation for the node positions. In case the localization problem has multiple solutions, the method gives an estimation which is very close to one solution in the set of all solutions of the problem.

If we increase the number of links in a random network keeping the number of nodes fixed then the probability that the network become uniquely localizable increases. The accuracy in position estimation may be better for networks having more edges. In the following we give some instances which show that as the number of edges increases in networks the position estimations become more accurate. 

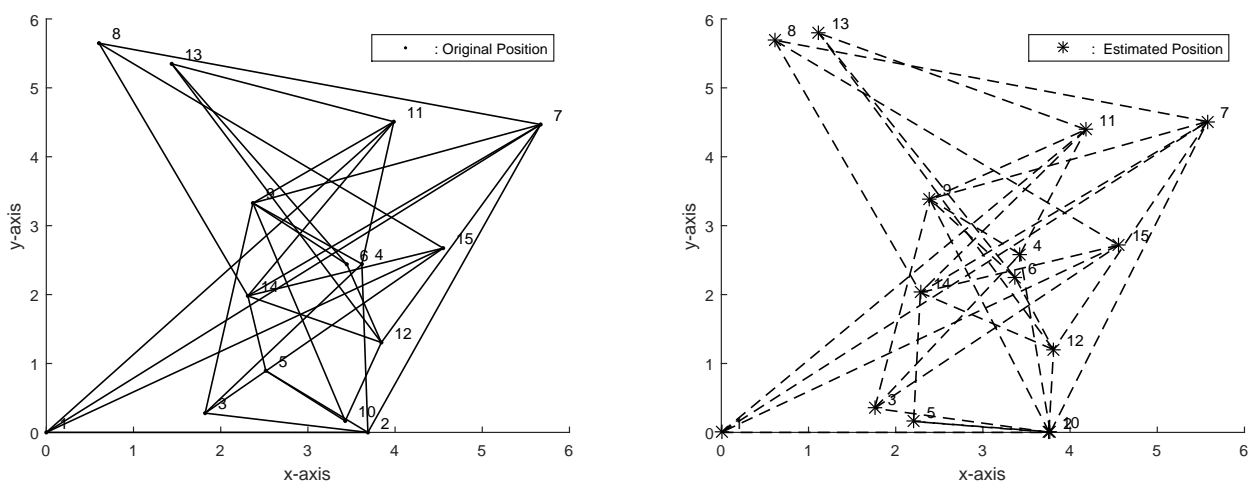

Figure 4: The original and estimated node positions of an uniquely localizable network

\subsection{Performance of the proposed method for increasing number of edges in a network}

Let $\aleph$ be a network having $n$ nodes and $e$ communication links. We define the network-density $\rho(\aleph)$ as the ratio of $e$ to the maximum possible number of edges $n(n-1) / 2$ (i.e., $\rho(\aleph)=2 * e / n(n-1)$ ). Any uniquely localizable network $\aleph$ with $n$ number of nodes lying in the plane has at least $2 n-2$ edges [18] (e.g., a Laman graph with an additional edge [21, a cycle bridge [27]). Thus if $\aleph$ is uniquely localizable $\rho(\aleph) \geq 2(2 n-2) / n(n-1)=4 / n$. It may be noted that $\rho(\aleph) \geq 4 / n$ does not imply the unique localizability of a network. In the following examples we have shown that the accuracy in position estimation increases with increasing network densities for randomly deployed networks as well the uniquely localizable networks.

Some arbitrarily networks with different densities In Figure [5, Figure 6] and Figure 7 we plot the original and estimated node positions for randomly deployed networks for three different network-densities $0.30,0.43$ and 0.52 . A small circle denotes the original position of a node and a star-mark denotes the computed position of a node. The error offset between the exact and estimated positions for individual nodes are indicated by lines. In these figures as the network-density increases the estimations for node locations become more accurate. It may be noted that large fluctuations occur due to flip ambiguities. Such fluctuations are also removed along with increasing densities.
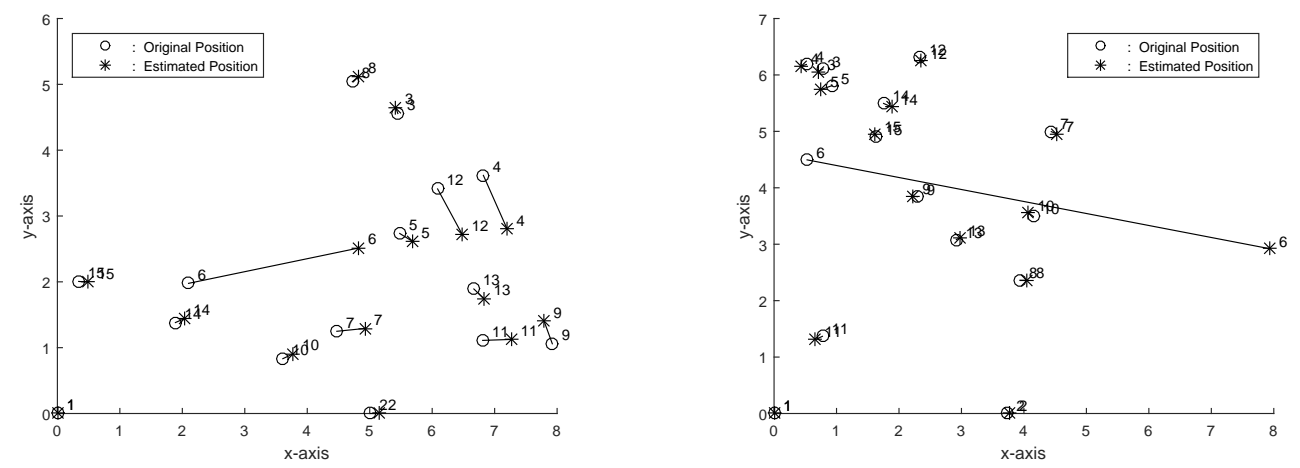

Figure 5: Random Network with density $=0.30$ Figure 6: Random Network with density $=0.43$

Some uniquely localizable networks with different network densities Figure 8 and Figure 9 show scenarios of uniquely localizable networks with three network-densities $0.30,0.43$ and 0.52. For each network-density, the estimated node positions for the underlying network are very 


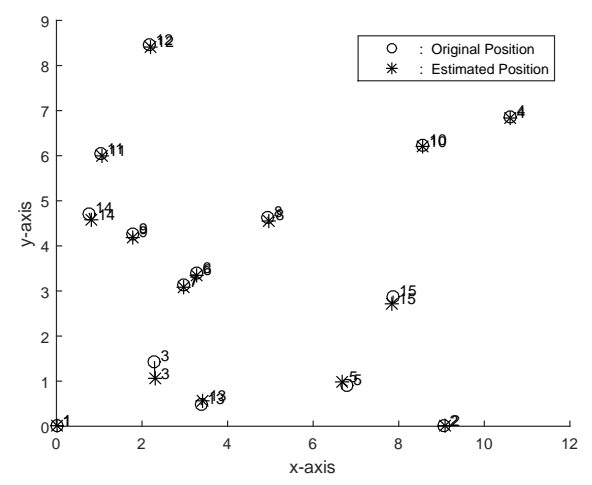

Figure 7: Random Network with density $=0.52$

close to their respective original positions. Again this difference between the uniquely localizable
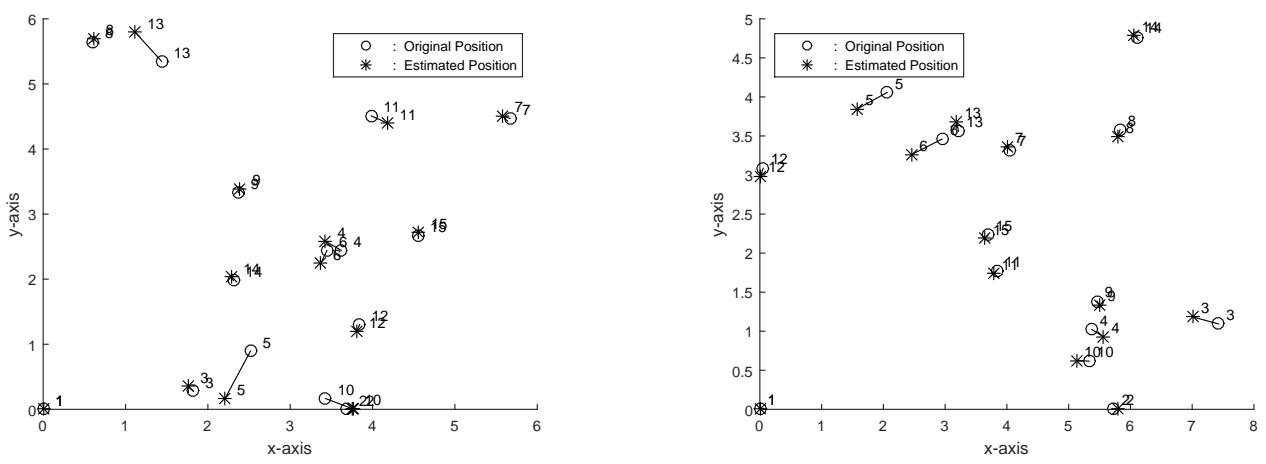

Figure 8: Original and estimated node positions for uniquely localizable network with network densities 0.30 and 0.43 from left to right

networks and randomly chosen networks occurs due to flip of vertices in randomly chosen networks. However from Figure 8 and Figure 9 it is clear that the proposed algorithm works well.

Observation2. Either the network is uniquely localizable or not the amount of errors are decreasing with increasing network densities.

Using an extensive number of networks, we shortly see that our claim is satisfied for a large number of networks.

\section{Error analysis of the root finding method}

We have seen in Section 5 that for a root $c_{0}=c_{0}^{*}$ of the function $\psi\left(c_{0}\right)-1$, the node positions for the underlying network is given by $x^{*}$ corresponding to which $\psi\left(c_{0}^{*}\right)=\mathbb{L}\left(x^{*}, c_{0}^{*}\right)=1$. To analyze the performance of the location estimation different researchers have considered different error metrics 8 , 12. In this work, we consider two different error metrics which are simple and standard. The performance of the root finding method is characterized with respect to these metrics.

\subsection{Error metrics}

1. Mean Error: The average of the differences between the estimated node positions (say $x_{i}^{\prime}$ ) and the original node position ( say $x_{i}$ ) for all nodes is the mean error of the position estimation, 


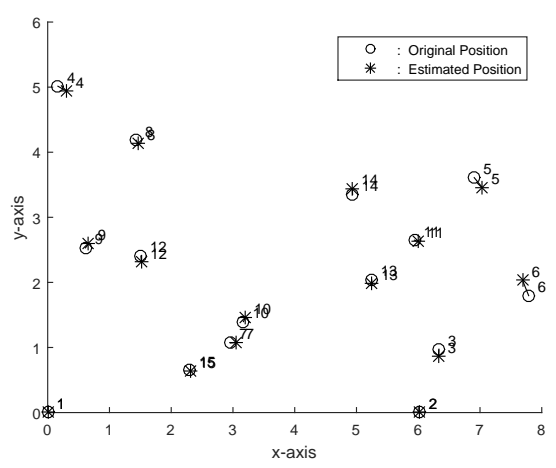

Figure 9: Original and estimated node positions for uniquely localizable network with network density $=0.52$

i.e., if a network has $n$ nodes then

$$
\text { Mean Error }=\frac{1}{n} \sum_{i}\left\|x_{i}-x_{i}^{\prime}\right\| .
$$

The mean error gives an overall idea about the accuracy of position estimations of the nodes in a network. If for a network the mean error is small enough then it can be said that the estimated node positions are very close to their original positions.

2. Maximum Error: This is the maximum difference among all the differences between the estimated node locations and the general node locations of a network, i.e.,

$$
\text { Max Error }=\max _{i}\left\|x_{i}-x_{i}^{\prime}\right\| .
$$

The worst fluctuation in node position estimation is measured by the maximum error in any case.

We consider a large number of random networks for finding positions of nodes using the root finding method. For each network we compute the mean and maximum errors in position estimation not removing the flip ambiguities. The error analysis shows that for this large class of networks, both the average mean and average maximum errors are decreasing with the growing network densities and gradually diminish to zero.

\subsection{Setting the Environment for executing our algorithm}

We select a rectangular region $(10$ unit $\times 10$ unit $)$ in the plane as the field of interest. Networks are randomly deployed within this region. We construct the graphs underlying to each network where the vertices and edges of the graph are considered as the nodes and communication links in the network respectively. The upper and lower bounds of the exact distance for each communication link are decided with a random distance error chosen within $0 \%$ to $15 \%$. The edge-weighted graph $G=(V, E, d)$ is constructed by setting the bounds as edge weights of the graph. The reconstruction of the network (i.e., assigning positions to the nodes) is carried out as follows:

- A region $K$ in $\mathbb{R}^{2 n}$ is considered (as described in Section 4). As discussed earlier all the feasible solutions (up to congruence) of the network localization problem have to be included in $K$.

- In $\mathbb{R}^{+}$(set of all positive real numbers) an interval is identified (Section 4) within which the function $\psi\left(c_{0}\right)-1$ must have a root.

- A point $x_{0}$ is randomly chosen from $K$ as the initial input for starting the SmoothingGrADIENTALGORITHM. 
- The estimated node positions of each network is recorded and compared to the original node positions to observe the accuracy of estimation.

\subsection{Performance of our algorithm}

We choose some network densities such that the underlying networks may be sparse or may be dense for these densities. For each network density, more than 500 networks are considered and positions of nodes are estimated using the proposed technique. From the original and estimated node positions of each network both the mean errors and maximum errors are computed. The average of all the mean errors and the average maximum errors for the selected network densities are computed.

We give two different scenarios for both the average mean error and the average maximum error on the basis of the amount of noise we allow in the input distance information. In the table 10 the average errors are recorded for random networks where we allow maximum $10 \%$ noise in the distance information. The computed average errors are small in this case. The Figure 11 associated to the table shows that, as the network density increases the average mean error as well the average maximum error decrease. We observe for higher network densities that the average mean errors are insignificant. Thus for randomly chosen networks having $10 \%$ noise in the distance information our proposed method gives a satisfactory result. We reach to a similar conclusion for the networks (Table 12 and the corresponding plot in Figure 13) where the average errors are computed for networks having $15 \%$ noise in distance information.

\begin{tabular}{|c|c|c|}
\hline$\rho(\aleph)$ & Mean error & Maximum error \\
\hline 0.40 & 1.4863 & 4.3133 \\
\hline 0.44 & 0.7368 & 2.3716 \\
\hline 0.51 & 0.3380 & 0.9376 \\
\hline 0.58 & 0.2856 & 0.8061 \\
\hline 0.64 & 0.1570 & 0.3563 \\
\hline 0.71 & 0.1301 & 0.3196 \\
\hline 0.78 & 0.0919 & 0.2008 \\
\hline 0.84 & 0.0820 & 0.1753 \\
\hline 0.91 & 0.0781 & 0.1730 \\
\hline
\end{tabular}

Figure 10: Errors computed for networks having maximum $10 \%$ noise in distance constraints

\begin{tabular}{|c|c|c|}
\hline$\rho(\aleph)$ & Mean error & Maximum error \\
\hline 0.47 & 0.8341 & 2.7959 \\
\hline 0.51 & 0.5798 & 1.5613 \\
\hline 0.56 & 0.3578 & 1.1638 \\
\hline 0.60 & 0.2672 & 0.7396 \\
\hline 0.64 & 0.2659 & 0.7014 \\
\hline 0.69 & 0.1810 & 0.4455 \\
\hline 0.73 & 0.1668 & 0.3875 \\
\hline 0.78 & 0.1312 & 0.3182 \\
\hline 0.82 & 0.1145 & 0.2588 \\
\hline
\end{tabular}

Figure 12: Errors computed for networks having maximum $15 \%$ noise in distance constraints

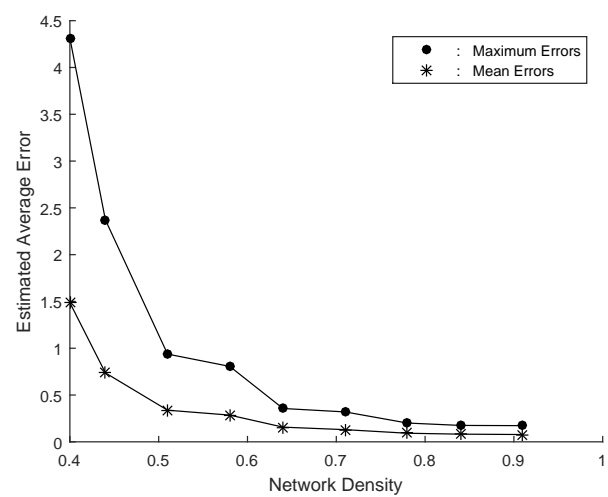

Figure 11: Polygon representing the values given in Table 10

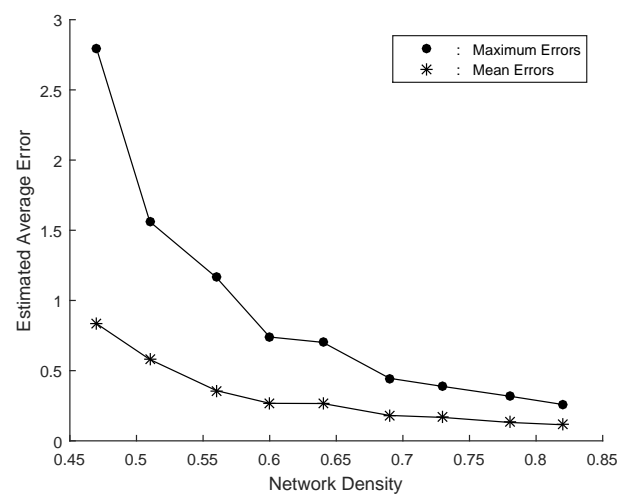

Figure 13: Polygon representing the values in Table 12 


\subsection{The advantages of the proposed root finding method}

In the literature, there are several network localization techniques which claim to solve the localization problem. We have mentioned some of the popular localization techniques in Section 1 which instead of solving the general network localization problem, have solved some modified version of it. Such modifications of the general network localization problem were done for implementing the existing convex optimization techniques like SDP, least square approximation etc. to solve the problem. For instances, in 12 the non-convex distance constraints are eliminated and solved using SDP. In [8], the non-convex constrains are relaxed such that the modified problem becomes convex optimization problem. In our work, we solve the general network localization problem (Problem 1) as the root finding problem (Problem 5 ). We enlist below some advantages of using the root finding method for solving the network localization problem.

1. The root finding method uses nonlinear non-convex Lagrangian optimization technique to solve the general network localization problem which considers both the convex and non-convex distance constraints without any modification or relaxation.

2. The actual feasible region of the network localization problem was modified in [8] for implementing the SDP. Therefore the errors in position estimation was due to incorrect constraints. On the contrary, in the proposed method the constraints are used without any modification. Therefore the errors in position estimation occur due to the inherent limitations of numerical root finding technique. By increasing the number of iteration of the root computations, one can achieve a desired level of accuracy in the position estimation.

3. The localization problems considered in 8 contains $0 \%$ to $10 \%$ noise over the actual distance measurements. The localization technique in [8] requires further improvement if the noise raises to $10 \%$ to $20 \%$ in distances. In reality, the distances constraints in localization problem mostly involves larger errors than the 10\%. In a test bed with 100 nodes we experience at most $50 \%$ noise in distance measurements. Though the examples exhibited in this paper contain maximum $20 \%$ noise in distance measurements, the proposed method may be used for computing node positions if the percentage of noise increases.

4. The convergence of root finding method is guaranteed to the unique solution of the network localization problem if the network is uniquely localizable. If the network is not uniquely localizable the method converges to one of the possible solutions of it.

\section{Conclusion}

The growing functionality of wireless sensor networks (WSN) in different fields of real life applications requires the actual node positions as an information for properly monitoring over the detected events. The existing techniques for solving the network localization problem do not solve the network localization problem. Instead, some variants of the problem have been solved so far. To the best of our knowledge, this is the first approach for solving the general network localization problem in noisy environment. We take the advantage of the nonlinear Lagrangian function for non-convex constraints to transform the general network localization problem to a root finding problem and use a simple iterative method for finding the roots. The method is guaranteed to converge to a solution. The node positions of any network can be computed up to a desired level of accuracy in this method. The examples show that for randomly deployed networks the root finding method gives good estimations for node positions.

We have a plan to validate the method with a test bed consisting more than 100 Arduino nodes. Our future target is to improve the root finding method in distributed techniques to enhance its performance in WSN. 


\section{ACKNOWLEDGMENT}

The first author would like to acknowledge Council of Scientific and Industrial Research (CSIR), Government of India, for giving the financial support in the form of Junior research fellowship to carry out this work.

\section{References}

[1] https://sites.math.washington.edu/ burke/crs/408/notes/nlp/line.pdf.

[2] M.A. ABRAMSON and C. AUDET. Convergence of mesh adaptive direct search to secondorder stationary points. SIAM J. OPTIM., 17(2):606619, 2006.

[3] I.F. Akyildiz, D. Pompili, and T. Melodia. Underwater acoustic sensor networks: research challenges. Ad Hoc Networks, 3:257279, May 2005.

[4] M.T. Apostal. Mathematical Analysis. Addison-wesley publisher, september 1973.

[5] B.G. Artner and J. Matousek. Approximation Algorithms and Semidefinite Programming. Springer-Verlag Berlin Heidelberg, 2012.

[6] J. Aspnes, T. Eren, D.K. Goldenberg, A.S. Morse, W. Whiteley, Y.R. Yang, B.D.O. Anderson, and P.N. Belhumeur. A theory of network localization. Mobile Computing, IEEE Transactions on, 5(12):1663-1678, Dec 2006.

[7] J. Aspnes, D. Goldenberg, and Y.R. Yang. On the computational complexity of sensor network localization. Algorithmic Aspects of Wireless Sensor Networks, pages 32-44, July 2004.

[8] P. Biswas, K.C. Toh, and Yinyu Ye. A distributed sdp approach for large-scale noisy anchorfree graph realization with applications to molecular conformation. SIAM Journal on Scientific Computing archive, 30:1251-1277, march 2008.

[9] B. Buchli, F. Sutton, and J. Beutel. Gps-equipped wireless sensor network node for highaccuracy positioning applications. Wireless Sensor Networks, 7158, 2012.

[10] F. H. Clarke. Optimization and Nonsmooth Analysis. 1983.

[11] Panos M. Pardalos Ding-Zhu Du. Nonconvex Optimization and its applications Minimax and Applications. 1995.

[12] L. Doherty, K.S.J. pister, and L. El Ghaoui. Convex position estimation in wireless sensor networks. In Proceedings of Twentieth Annual Joint Conference of the IEEE Computer and Communications Societies, pages 1655-1663, 2001.

[13] T. Fujie and M. Kojima. Semidefinite programming relaxation for nonconvex quadratic programs. Journal of Global optimization, (10):367380, 1997.

[14] T. Fujie and M. Kojima. Semidefinite programming relaxation for nonconvex quadratic programs. Journal of Global Optimization, 10:367-380, June 1997.

[15] C.J. Goh and X.Q. Yang. A nonlinear lagrangian theory for non-convex optimization. Optimization theory and Applications, 109:99-121, april 2001.

[16] Meredith J. Goldsmith. Sequential quadratic programming methods based on indefinite hessian approximations. Diss. stanford university, March 1999.

[17] M. Hefeeda and M. Bagheri. Wireless sensor networks for early detection of forest fires. In Mobile Adhoc and Sensor Systems, 200\%. MASS 200\%. IEEE International Conference on, pages 1-6, Oct 2007. 
[18] B. Jackson and T. Jordán. Connected rigidity matroids and unique realizations of graphs. Journal of Combinatorial Theory Series B, 94(1):1-29, 2005.

[19] Fidan B. Kannan A.A. and Mao G. Analysis of flip ambiguities for robust sensor network localization. IEEE Transactions on Vehicular Technology, 59(4):2057-2070, May 2010.

[20] K. Khedo1, R. Perseedoss, and A. Mungur. A wireless sensor network air pollution monitoring system. International journal of Wireless and mobile networks, 2(2), may 2010.

[21] G. Laman. On graphs and rigidity of plane skeletal structures. Journal of Engineering Mathematics, 4(4), dec 1970.

[22] Du Pang, Ju. Global convergence methods for nonsmooth equations with finitely maximal functions and their applications. Journal of Applied Mathematics and Informatics, 32(5-6):609 619, May 2014.

[23] M.V. Ramesh. Real-time wireless sensor network for landslide detection. In Sensor Technologies and Applications, pages 405-409, June 2009.

[24] A. Saha and B. Sau. Network localization with noisy distances by non-convex optimization. In Proceedings of WiSPNET'16 (The international Conference on Wireless Communications, Signal Processing and Networking), 2016.

[25] Ananya Saha and Buddhadeb Sau. Network localization by non-convex optimization. In $A C M$ MobiMWareHN'17 Workshop to be held in conjunction with 18th ACM MobiHoc 2017, Chennai, India, july.

[26] B. Sau and K. Mukhopadhyaya. Length-based anchor-free localization in a fully covered sensor network. In Proc. of the First international conference on COMmunication Systems And NETworks, COMSNETS'09, pages 137-146, Piscataway, NJ, USA, 2009. IEEE Press.

[27] B. Sau and K. Mukhopadhyaya. Localizability of wireless sensor networks: Beyond wheel extension. In SSS, volume 8255 of $L N C S$, pages 326-340. Springer, 2013.

[28] B. Sau, S. Mukhopadhyaya, and K. Mukhopadhyaya. Localization control to locate mobile sensors. In Proc. of the Third international conference on Distributed Computing and Internet Technology, ICDCIT'06, pages 81-88, Berlin, Heidelberg, 2006. Springer-Verlag.

[29] James B. Saxe. Embeddability of weighted graphs in k-space is strongly np-hard. In Proceedings of the 17th Allerton Conference on Communication, Control and Computing, pages 480-489, 1979.

[30] L. Vandenberghet and S. Boyd. Semidefinite programming. SIAM Review, 38:49-95, March 1996.

[31] Chen X. and Zhou W. Smoothing nonlinear conjugate gradient method for image restoration using nonsmooth nonconvex minimization. SIAM Journal on Imaging Sciences, 3(4):765-790, October 2010.

\section{A Clarkr stationary point}

In non-smooth analysis F.H. Clarkr introduces the notion of generalized derivatives, normal and tangent cones [10] to grapple the non-smoothness together with the absence of convexity. Let $f: B \rightarrow \mathbb{R} \cup \infty$ be a non-smooth locally Lipschitz function where $B$ is a Banach Space. Let $B^{*}$ be the dual space of $X$. 
Definition 3. [10] The generalized directional derivative of $f$ at a point $\tilde{x}$ and in the direction of the vector $v$ is defined as

$$
f^{0}(\tilde{x}, v)=\limsup _{y \rightarrow x, t \downarrow 0} \frac{f(y+t v)-f(y)}{t} .
$$

Using theorems of functional analysis [] it may be said that there is a linear functional $\zeta: B \rightarrow \mathbb{R}$ such that $f^{0}(x, v) \geq \zeta(v)$ for all $v \in B$. We adopt the convention of using $\langle\zeta, v\rangle$ for $\zeta(v)$.

Definition 4. The generalized gradient at a point $\tilde{x}$ of $f$ is defined as

$$
\partial f(\tilde{x}):=\left\{\zeta \in B^{*}: f^{0}(\tilde{x}, v) \geq\langle\zeta, v\rangle\right\}
$$

for all $v \in B$.

Remark: The function $f$ is strictly differntiable at a point $\tilde{x}$ if $\partial f(\tilde{x})$ is a singleton set, i.e., $\partial f(\tilde{x})=\{\nabla f(\tilde{x})\}$.

Theorem 7. $\partial f(\tilde{x})$ can be proved to be non-empty, convex and weak*-compact subset of $B^{*}$.

The norm $\|\zeta\|_{*}$ in $B^{*}$ is defined as

$$
\|\zeta\|_{*}=\sup \{\langle\zeta, v\rangle \mid v \in B,\|v\| \leq 1\} .
$$

Definition 5. [2] A point $\tilde{x}$ is said to be a clarkr stationary point of $f$ if $0 \in \partial f(\tilde{x}, v)$, i.e., $f^{0}(\tilde{x}, v) \geq 0$ for every direction vector $v$ in the clarkr tangent cone.

Definition 6. [10] The clarkr tangent cone to a point $\tilde{x} \in U \subset X$ consists of all the vectors $v$ of $X$ for which the following condition holds:

For every sequence $\left\{x_{i}\right\}$ in $U$ converging to $\tilde{x}$ and sequence $\left\{t_{i}\right\}\left(t_{i}>0 \forall i\right)$ decreasing to 0 , there is a sequence $\left\{v_{i}\right\}$ in $X$ converging to $v$ such that $x_{i}+t_{i} v_{i} \in U$ for all $i$.

The following theorems are useful for finding the clarkr stationary points of a non-smooth function.

Theorem 8. [10] $\zeta \in \partial f(\tilde{x})$ if and only if $f^{0}(\tilde{x}, v) \geq\langle\zeta, v\rangle$ for all directions $v$ in the underlying space.

Theorem 9. [10] Let $\nabla f(\tilde{x})$ exist at a point $\tilde{x}$ near which $f$ is Lipschitz continuous. Then $\nabla f(\tilde{x}) \in$ $\partial f(\tilde{x})$ 